\title{
Verwaltung ohne civitas? \\ Zur administrativen Organisation zwischen Vinxtbach und Nahe am Mittelrhein.
}

\author{
Elena Köstner
}

„Gallias et Hispanias provincias, item Germaniam qua includit Oceanus a Gadibus ad ostium Albis fluminis pacavi. " Mit diesen Worten beschreibt Augustus u.a. sein Wirken in Gallien und Germanien und postuliert den Abschluss erfolgreicher Operationen. Auch die Etablierung grundlegender Verwaltungsstrukturen entlang des Rheins, der civitates, fällt in augusteisch-tiberische Zeit. Dazu zählen u.a. die civitas der Treverer, der Ubier, der Vangionen und der Nemeter. Doch fehlen derartige Strukturen im Raum zwischen Vinxtbach und Nahe am Mittelrhein. Dieses Gebiet gehörte vormals zum Stammesgebiet der Treverer. Nach Caesars Sieg über die Treverer und der Neuorganisation innerhalb Galliens unter Augustus wurde dieser Raum ager publicus und somit Eigentum des römischen Staates. Aber auch in den nachfolgenden Jahrzehnten und Jahrhunderten wurden hier keine civitates eingerichtet. „So kennen wir z.B. im nordwestlichen Obergermanien links des Rheins, d.h. nördlich der civitas Vangionum [...] und der Caeracaten [...] und südlich der Ubier bzw. der colonia Claudia Ara Agrippinensium in der hohen Kaiserzeit keine einzige civitas. “2 ${ }^{2}$ Das sieht auch Raepsaet-Charlier so: ,[...] son existence [= civitas] ne parait pas faire de donte mais sa dénomination et sa capitale au moins font difficulté $[\ldots] .{ }^{، 3}$

Doch wie wurde dann in diesen Gebieten Verwaltungsarbeit betrieben und auf welche Strukturen gründete sich diese? In der Forschung wurde und wird bislang angenommen, dass die Verwaltung über die Pachtwirtschaft der Domänen und ihre Großgrundbesitzer erfolgte. ${ }^{4}$ Raepsaet-Charlier hält eine Verwaltung über kaiserliche Domänen ohne civitas-Strukturen für eine „hypthèse qui nous parait peu satisfaisante notament car elle supposerait soit des vici ,autonomes ${ }^{6}$ soit sous l'autorité militaire. ${ }^{65}$ Doch wären für die Bedürfnisse der römischen Provinzialverwaltung - Erhebung von Steuern und Abgaben, Rekrutierung von Soldaten, etc. - m.E. Zentralorte von Nöten, die wiederum ein sie umgebendes Umland kontrollierten. Am Beispiel des territorium metallum von Mayen, einem Distrikt im ager publicus zwischen Vinxtbach und Nahe, der sich seit der frühen Kaiserzeit auf den Bergbau gründete und in dem seit spätrömischer Zeit auch die Keramikproduktion eine wichtige Rolle spielte, können administrative Strukturen aufgezeigt werden. Das territorium metallum von Mayen dient somit exemplarisch der Darstellung der administrativen Organisation im ager publicus zwischen Vinxtbach und Nahe.

\section{Die Verhältnisse am Mittelrhein nach dem gallischen Krieg}

In vorrömischer Zeit erstreckte sich das Stammesgebiet der Treverer zwischen Rhein und Maas. Während Caesars Feldzüge lediglich den römischen Machtanspruch in Gallien bekundeten, fand eine administrative Strukturierung der eroberten Gebiete

\footnotetext{
${ }^{1}$ R. Gest. div. Aug. 26, 2.

${ }^{2}$ VitTINGHOFF 1994A, 74.

${ }^{3}$ RAEPSAET-CHARLIER 1999, 292.

${ }^{4}$ CÜPPERS 1990, $110 f$.

${ }^{5}$ RAEPSAET-CHARLIER 1999, 312.
} 
erst nach 27 v. Chr. durch Augustus statt. „Caesar [Anmerkung: Augustus] hingegen hatte inne den Rest von Spanien - das heißt das Gebiet um Tarraco und Lusitanien und ganz Gallien - nämlich Gallia Narbonensis, Gallia Lugdunensis, Aquitania und Belgica, die Einwohner sowohl wie die unter ihnen lebenden Fremden. "6 Die Provinz Gallia Belgica galt zu diesem Zeitpunkt noch nicht als befriedet und wurde von einem legatus Augusti pro praetore verwaltet. Nach dem gallischen Krieg wurden Teile des Stammesgebietes der Treverer entlang des Rheins abgetrennt und eine römische civitas Treverorum gegründet. Dies geschah wahrscheinlich zwischen $27 \mathrm{v}$. und 16/17 n. Chr. Einen Hinweis auf diesen Vorgang liefert ein Grabstein aus Mainz-Weisenau: „Respectus / nat(ione) Tre(ver) an/nor(um) VIII / Veranius $5 /$ nat(ione) Trev(er) / anno(rum) III fil(ii) / Samocna / nat(ione) Tre(vera) an/nor(um) II filia ${ }^{10} / \operatorname{Pr}$ (a)esens / pater fili(i)s / suis posuit / ob pieta(tem) / h(ic) s(iti) sunt. " ${ }^{77}$ Die (Teil-)Stämme der Aresaces und Caeracates siedelten südlich bzw. südöstlich von Mogontiacum/Mainz. ${ }^{8}$ Die Nennung der Zugehörigkeit zum Stamm der Treverer macht nach Witteyer nur dann Sinn, wenn dieser Grabstein außerhalb des Treverergebiets stand. ${ }^{9}$ Da der Grabstein in das frühe 1. Jahrhundert $\mathrm{n}$. Chr. datiert, kann daraus geschlossen werden, dass es zu einer Gebietsabtrennung gekommen war, die zum Zeitpunkt der Aufstellung des Grabsteins wohl bereits vollzogen war. Würde sich der Grabstein zum Zeitpunkt der Aufstellung auf Treverergebiet befunden haben, wäre eine Nennung der Treverer wohl redundant. Ein weiterer Hinweis, der für die Gebietsabtrennung in dem oben genannten Zeitrahmen spricht, ist der Aufenthalt des Augustus in Gallien zwischen 16 und 13 v. Chr., währenddessen er u.a. die Verlegung von Truppen aus dem Inneren Galliens an den Rhein, einen Zensus und die Neuordnung der Provinzen veranlasst haben soll. ${ }^{10}$ Auch die territorialen Veränderungen, von denen die Treverer betroffen waren, könnten in diesem Zeitfenster beschlossen und eventuell durchgeführt worden sein.

Doch erst unter Domitian wurden die beiden germanischen Provinzen gegründet: Während das Militärdiplom CIL XVI 28 (82 n. Chr.) diese Region am Rhein lediglich als Germania bezeichnet und damit von einem Distrikt spricht, nennt CIL XVI 36 (90 n. Chr.) die Germania superior und geht somit von zwei Provinzen aus. ${ }^{11}$ Demnach kann die Aufteilung und Gründung der beiden germanischen Provinzen für diesen Zeitraum, eventuell nach Domitians Feldzügen gegen die Chatten (83 bis $85 \mathrm{n}$. Chr.), angenommen werden. ${ }^{12}$ Auch zu diesem Zeitpunkt gibt es keine Hinweise dafür, dass zwischen Vinxtbach und Nahe civitates etabliert wurden. Dieser Raum blieb somit ager publicus. ${ }^{13}$

\footnotetext{
${ }^{6}$ Cass. Dio 53,12,5.

${ }^{7}$ CIL XIII 11888=AE 1913, 130=CSIR II 6,34 (frühes 1. Jahrhundert n. Chr., vermutlich tiberische Zeit); WITTEYER 1999, 1022.

${ }^{8}$ Zu Caeracates: Tac. hist. 4,70,3; AE 1965, 247; zu Aresaces: CIL XIII 7252; CIL XIII 11825; AE 1929, 173.

${ }^{9}$ WITTEYER 1999, $1022 \mathrm{f}$.

${ }^{10}$ Vell. 2,97,1.

${ }^{11}$ Im Gegensatz dazu u. a.: AUSBÜTTEL 2011, 392-410; ECK 2009, 188-195.

${ }^{12}$ Suet. Dom. 6,2: „Bellum civile motum a L. Antonio, superioris Germaniae praeside, confecit absens felicitate mira, cum ipsa dimicationis hora resolutus repente Rhenus transituras ad Antonium copias barbarorum inhibuisset. De qua victoria praesagiis prius quam nuntiis comperit, siquidem ipso quo dimicatum erat die statuam eius Romae insignis aquila circumplexa pinnis clangores laetissimos edidit; pauloque post occisum Antonium adeo vulgatum est, ut caput quoque adportatum eius vidisse se plerique contenderent. “

${ }^{13}$ Unter ager publicus versteht man u.a. die Ländereien, die durch Konfiskationen in den Territorien besiegter Ethnien den Besitz des römischen Staates erweiterten. Wurde der ager publicus niemandem mittels Pacht beispielsweise - zugewiesen, so wurde er gemeinsam mit dem anderen staatlichen Eigen-
} 


\section{Die Dimensionen des ager publicus am Mittelrhein}

Um auf die Dimensionen des civitas-freien Raumes am Mittelrhein schließen zu können, sollen zunächst die dieses Gebiet direkt umgebenden Territorien und ihre Ausmaße betrachtet werden. Im Mainzer Raum siedelten in vorrömischer Zeit Aresaces und Caeracates, die Witteyer in der, ,äußersten Randzone des treverischen Stammesgebietes“ verortet. ${ }^{14}$ Das Siedlungsgeschehen veränderte sich in augusteischer Zeit: Um 13/12 v. Chr. wurden Militärlager in Mogontiacum/Mainz errichtet. ${ }^{15}$ Damit wurde das diesen Militärstandort umgebende Land zu prata legionis und unterstand der Verwaltung der Streitkräfte. ${ }^{16}$ Eine civitas wurde hier in der frühen Kaiserzeit nicht gegründet. ${ }^{17}$ Aufgrund der Tatsache, dass in der Spätantike die Hauptorte der kaiserzeitlichen civitates zu Bischofssitzen wurden und von diesen aus die Diözesen verwaltet wurden, stimmen diese im Wesentlichen mit dem Gebiet der civitates aus römischer Zeit überein. ${ }^{18}$ Bingium/Bingen und Bad Kreuznach gehörten zur Mainzer Diözese und waren demnach nicht mehr Teil des ager publicus am Mittelrhein. ${ }^{19}$ Wahrscheinlich bildete die Nahe die Grenze zwischen den Diözesen Mainz und Trier. ${ }^{20}$ Südlich von Mainz entlang des Rheins entstanden in augusteisch-tiberischer Zeit neue civitates - u.a. die der Vangionen, Nemeter und Tribocer. ${ }^{21}$

Derartige Vorgänge sind nicht nur südlich von Mogontiacum/Mainz feststellbar, sondern auch nördlich des Vinxtbaches. Dieser Wasserlauf, der zwischen Bad Breisig und Brohl in den Rhein mündet, stellt nicht nur die südliche Grenze der Germania inferior dar, sondern ist gleichzeitig auch die Südgrenze der civitas Ubiorum. ${ }^{22}$ Der Name Vinxtbach kann vom lateinischen ad fines abgeleitet werden und verweist auf die Grenzsituation: „Der Teil des Landes am Rhein von der See bis zum Flusse Obrincas heißt Germania inferior. “23 Auch Weihinschriften für Grenzgötter belegen dies: „Finibus et / genio loci / I(ovi) O(ptimo) M(aximo) milit(es) / leg(ionis) XXX V(lpiae) V(ictricis) ${ }^{5} /$ M(arcus) Massiani/us Secundus et T(itus) Aurelius / Dosso / $v$ (otum) s(olverunt) l(ibentes) m(erito). ${ }^{24} \mathrm{Zu}$ den Ausmaßen der civitas Ubiorum äußert sich Tacitus: „Ingressis Novaesium XVI legio coniungitur. Additus Voculae in partem curarum Herennius Gallus legatus; nec ausi ad hostem pergere <ad tertium

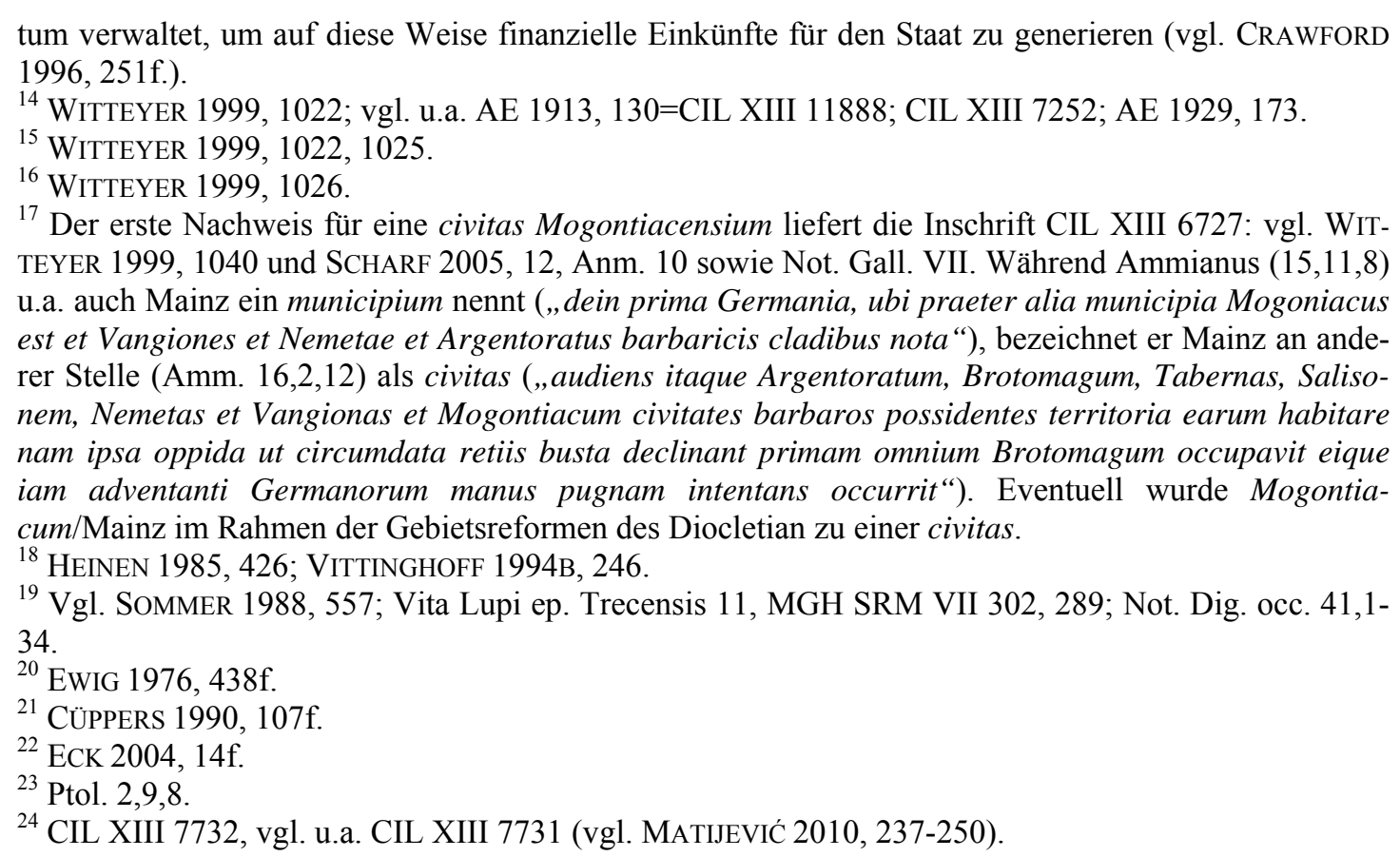
rer Stelle (Amm. 16,2,12) als civitas („,audiens itaque Argentoratum, Brotomagum, Tabernas, Salisonem, Nemetas et Vangionas et Mogontiacum civitates barbaros possidentes territoria earum habitare nam ipsa oppida ut circumdata retiis busta declinant primam omnium Brotomagum occupavit eique iam adventanti Germanorum manus pugnam intentans occurrit"). Eventuell wurde Mogontiacum/Mainz im Rahmen der Gebietsreformen des Diocletian zu einer civitas.

${ }^{18}$ Heinen 1985, 426; VitTinghofF 1994B, 246.

${ }^{19}$ Vgl. Sommer 1988, 557; Vita Lupi ep. Trecensis 11, MGH SRM VII 302, 289; Not. Dig. occ. 41,134.

${ }^{20}$ EwIG 1976, $438 f$.

${ }^{21}$ CÜPPERS 1990, $107 \mathrm{f}$.

${ }^{22}$ ECK 2004, 14 f.

${ }^{23}$ Ptol. 2,9,8.

${ }^{24}$ CIL XIII 7732, vgl. u.a. CIL XIII 7731 (vgl. MATIJEVIĆ 2010, 237-250). 
decumum ab Novaesio lapidem> (loco Gelduba nomen est) castra fecere. “25 An die civitas Ubiorum schlossen sich im Norden der Germania inferior weitere civitates an, die jedoch für die Rekonstruktion der Dimensionen des ager publicus zwischen Vinxtbach und Nahe keine Rolle spielen.

Wichtiger dafür ist jedoch die civitas Treverorum, die trotz der oben genannten Gebietsabtrennung bestehen blieb. ${ }^{26}$ Auch für die Grenze zwischen der civitas Ubiorum und der civitas Treverorum liefert die Einteilung der kirchlichen Diözesen wichtige Hinweise: Die Grenze zwischen den Diözesen Trier und Köln liegt beim vicus Ausana/Oos (südlich von Icorigium/Jünkerath) und entspricht nicht nur der Grenze zwischen der civitas Treverorum und der civitas Ubiorum, sondern auch derjenigen zwischen den Provinzen Gallia Belgica und Germania inferior. ${ }^{27}$

Weitere Hinweise auf das Fehlen von civitas-Strukturen im ager publicus liefern die Meilensteine. Laut Rathmann kann ,,von den Meilenzählungen auf die Territoriumsgröße und den damit verbundenen Verwaltungsbezirk“ geschlossen werden. ${ }^{28}$ Im Falle der civitas Treverorum stimmt die Meilenzählung nicht mit der Territoriumsgrenze überein. ${ }^{29}$ Die Zählung auf den Miliarien zwischen Augusta Treverorum/Trier und Mogontiacum/Mainz missachtete die Provinzgrenze zwischen der Gallia Belgica und der Germania superior, denn bis nach Mainz wurde von Trier aus gezählt: „Imp(eratori) Caes(ari) / T(ito) Aelio Ha/driano An/tonino Aug(usto) $5 /$ pio pont(ifici) $\max ($ imo) / tr(ibunicia) pot(estate) II co(n)s(uli) II / p(atri) p(atriae) a col(onia) Aug(usta) / Tr(everorum) m(ilia) p(assuum) LXXX/VIII. " "30 Auf der Rheintalstraße von Bingium/Bingen über Confluentes/Koblenz bis zur niedergermanischen Grenze wurde jedoch nach Mogontiacum/Mainz gezählt: „Perpetuo [imp(ertori) L(ucio)] / Domitio [Aure]/liano pi[o fel(ici)] / Aug(usto) p(ontifici) m(aximo)

\footnotetext{
25 Tac. hist. 4,26,3; vgl. ebs. Tac. hist. 4,79,1f.: „Namque et Civilis illuc intenderat, non invalidus, flagrantissima cohortium suarum integra, quae e Chaucis Frisiisque composita Tolbiaci in finibus Agrippinensium agebat.“ Tac. hist. 4,28,1f. „Caesar cohortes eorum in vico Marcodura incuriosius agentes, quia procul ripa aberant. “ Plinius ergänzt, dass die Nordgrenze der civitas nördlich von Gelduba/Gellep liegt (vgl. ebs. Strab. 4,3,4; Tac. Germ. 28,4; Tac. ann. 12,27,1). Außerdem erstreckte sich das Territorium der Ubier bis zur Rur bzw. bis zur Wurm bei Aachen, so EcK 2004, 16. Im Nordwesten verlief die Grenze zwischen der Colonia Claudia ara Agrippinensium/Köln und der Colonia Ulpia Traiana/Xanten vermutlich entlang der Orte Aachen - Erkelenz - Mönchengladbach - Gellep, so ECK 2004, $17 \mathrm{f}$.

${ }^{26}$ Das Territorium der Treverer in römischer Zeit kann wie folgt beschrieben werden: „Im Osten der Fluss Ließer und die Orte Oos, Jünkerath, Hillesheim, Pelm, Daun, Manderscheid, Wittlich, Platten, Rachtig, Wederath; im Norden der Oosbach und die Orte Billig, Rommersheim, Lünebach, Lichtenborn, Irrhausen, der Fluss Irsen bis zur Mündung in die Our bei Eisenbach, Bastogne, Cugnon a. d. Semois; im Westen nördlich von Carignan über die Chiers, östlich von Mouzon, dann entlang der Maas bis südlich von Stenay, zwischen Longuyon und Longwy entlang der Chiers; im Südwesten Kannerbach, Bistbach, Köllerbach; im Südosten Tholey, die Nahe von Birkenfeld bis Weierbach, der Fischbach.“(HEINEN 1985, 426).

${ }^{27}$ Marmagen und Gerolstein sind weitere Orte entlang dieser Grenze. Die Meilensteine aus diesem Gebiet zählen entweder ab der colonia Claudia ara Agrippinensium/Köln, der Hauptstadt der Germania inferior, oder ab Augusta Treverorum/Trier, dem Hauptort der civitas Treverorum. Der stark fragmentierte Meilenstein an der Straße Trier - Köln gibt die Entfernung zwischen colonia Claudia ara Agrippinensium und Marmagen mit 39 Meilen (58 Kilometern) an: „, [- - ] II co(n)s(uli) / a col(onia) Agripp(inensium oder Agrippina) [- - ] / m(ilia) p(assuum) XXXIX“ (CIL XIII 9136). Der Meilenstein, der zwischen Gerolstein und Prüm gefunden wurde, gibt die Distanz zwischen Prüm und Trier mit 22 Meilen (33 Kilometern) an: „Imp(eratori) Caes(ari) divi / Traiani Parthici / fili(o) divi Nervae / nepoti / Traiano Hadriano / Aug(usto) pontif(ici) max(imo) / tr(ibunicia) potest(ate) V co(n)s(uli) III p(atri) p(atriae) / a col(onia) Aug(usta) mil(ia) [p(assuum)]/ XXII“ (CIL XIII 9133).

${ }^{28}$ RATHMANN 2003, 113.

${ }^{29}$ RATHMANN 2033, 113.

${ }^{30}$ CIL XIII 9131; vgl. u.a. AE 1979, 417, AE 1979, 418.
}

http://www.fera-journal.eu 
t(ribunicia) [pot(estate)] 5/ co(n)s(uli) p(atri) p(atriae) $\operatorname{pr}[o c o(n) s(u l i)] \quad / \quad a$ Mog(ontiaco) / leugas XXV[---]." ${ }^{31}$ Das Stammesgebiet der Treverer reichte vor dem gallischen Krieg und z.T. auch noch in augusteischer Zeit bis zum Rhein. Die claudischen Meilensteine zählen von Trier aus und übergehen Mainz. ${ }^{32}$ Die Straße zwischen Trier und Mainz wurde frühestens unter Agrippa errichtet bzw. existierte spätestens in Zusammenhang mit der Germanienoffensive des Drusus seit 12 v. Chr. ${ }^{33}$ Anscheinend behielt man auch nach der Gründung der germanischen Provinzen Augusta Treverorum/Trier als caput viae bei; das belegen die oben genannten Meilensteine, die aus dem 2. und 3. Jahrhundert n. Chr. stammen. Aber innerhalb der germanischen Provinzen war auch Mogontiacum/Mainz ein caput viae. Die Zählweise auf den Miliarien belegt, dass zwischen Vinxtbach und Nahe keine civitas-Strukturen existierten, da sonst die civitas-Hauptorte als capita viarum auf den Meilensteinen zu finden wären. ${ }^{34}$ Dies unterstreicht die These, dass das Gebiet zwischen Vinxtbach und Nahe ager publicus war.

\section{Das territorium metallum von Mayen als Beispiel eines Verwaltungsdistrikts im ager publicus am Mittelrhein}

Es stellt sich die Frage, wie die Verwaltung im ager publicus am Mittelrhein gestaltet war. Erste Anhaltspunkte für die Gestaltung der administrativen Organisation der römischen Kaiserzeit liefert das territorium metallum von Mayen. ${ }^{35}$ Sowohl die lex metallis dicta (CIL II 5181) als auch die lex metalli Vipascensis (CIL II 1892) aus Vipasca (in Lusitania) geben dafür wichtige Hinweise. Die beiden leges unterscheiden zwischen vicus, metallum und territorium metallum. ${ }^{36}$ Während ein metallum auch Institutionen des Gemeinwesens miteinschloss und daher als administrativer Distrikt mit eigenen Gesetzen und eigenem umgebenden Land gesehen werden kann, handelt es sich bei territorium um einen juristischen Distrikt und somit um den Kompetenzbereich des Prokurators. ${ }^{37}$ Der vicus übernahm zentralörtliche Funktionen für den Distrikt und kann als Handels- und Wirtschaftszentrum sowie als verkehrstechnischer Knotenpunkt erachtet werden.

Auf die Verhältnisse Mayens angewendet, ergibt sich daher folgendes Szenario: Die wichtigsten Erzeugnisse aus dem vicus von Mayen waren „Mühlsteine aus Basaltlava, Bausteine aus Tuff und Gebrauchsgeschirr aus Ton““. ${ }^{38}$ Die Töpfereien

\footnotetext{
${ }^{31}$ CIL XVII 562; vgl. u.a. CIL XVII 564=XIII 9140, CIL XVII 567=XIII 9143.

${ }^{32}$ RATHMANN 2003, 114; SCHARF 2005, 12f.; vgl. Anm. 19.

${ }^{33}$ RATHMANN 2003, 114, Anm. 669.

${ }^{34}$ In diesem Zusammenhang stellt sich auch die Frage, wer für die cura viarum am Mittelrhein zuständig war, denn laut RATHMANN 2003, 114 lag die Verantwortung dafür nach der Gebietsabtrennung nicht mehr bei der civitas Treverorum.

${ }^{35}$ Eine ausführlichere Darstellung dieser Aspekte: KÖSTNER 2012, 73-85.

${ }^{36}$ Vgl. HIRT 2010, 49; CIL II 5181 II. 27-29; CIL II 5181 II. 32-35; CIL II 5181 II. 40f.; CIL II 1892 II. 1-9; CIL II 1892 II. 37f.; CIL II 1892 II. 47-57; CIL II 1892 II. 59-61.

${ }^{37}$ HIRT 2010, 49f. Ein metallum war gemäß den Digesten (7,19,2f.) ein Gebiet, in dem Steine, Metalle und andere Rohstoffe abgebaut werden. $\mathrm{Zu}$ den Aufgaben und Pflichten eines Prokurators: Tac. ann. 12,60,2f., Cass. Dio 53,15,3.

${ }^{38}$ SCHAAFF 2010, 265. In den Tuffsteinbrüchen im Tal des Krufter Baches und des Brohltals wurde mit dem Abbau am Anfang des 1. Jahrhunderts n. Chr. begonnen und das römische Militär übernahm diese Aufgabe (vgl. SchAAFF 2010, 268f.; SCHAAFF 2012, 10-14; Matijević 2010, 41-236; beispielsweise CIL XIII 7693-7712, CIL XIII 7714-7722, CIL XIII 7725-7727; AE 1922, 62; AE 1926, 20; AE 1926, 21; LEHNER 1921, 133f.). Hier existierte zunächst keine Pachtwirtschaft. Nach ca. 100/120 n. Chr. übernahmen diese Arbeiten private conductores, so RÖDER 1957, 229. Laut MATIJEVIĆ 2010, 194 ist die Annahme einer Privatisierung der ,Abbaugebiete in der östlichen Eifel ab der Mitte des 2. Jahrhun-
} 
befanden sich innerhalb des vicus-Areals. ${ }^{39}$ Einige Papyri aus Oxyrhynchus/AlBahnasa in Aegyptus liefern Hinweise auf die pachtwirtschaftlichen Strukturen im Töpfereiwesen. ${ }^{40}$ Die Pachtverträge beinhalteten folgende Aspekte: Den coloni wurden nicht nur Lagerräume, Brennöfen und weitere Werkzeuge zur Verfügung gestellt, sondern auch Tone, Holz, Wasser, Pech etc.; dafür erhielten die conductores vertraglich festgelegte Mengen an Keramikwaren. ${ }^{41}$ Sowohl die Basaltlavasteinbrüche bei Mayen als auch die Tuffsteinbrüche im Brohltal und im Tal des Krufter Baches gehörten demnach zu einem administrativen Bezirk mit festen Grenzen. ${ }^{42}$ Für die Produktion von Mühlsteinen aus Basaltlava und von Bausteinen aus Tuff bieten die lex Manciana und lex Hadriana (Africa Proconsularis) wichtige Anhaltspunkte. ${ }^{43}$ Da sich die Steinbrüche ebenso wie die Töpfereien des territorium metallum von Mayen im ager publicus befanden, war der römische Staat Eigentümer dieses Landes und seiner Ressourcen. Im Falle der Steinbrüche verpachtete dieser über die procuratores saltus an conductores. ${ }^{44}$ Der saltus wurde in Parzellen unterteilt und diese an coloni verpachtet. Die coloni entrichteten ihre Pachtabgaben an die conductores und über diese und die procuratores gelangten die erwirtschafteten Erträge in den fiscus der Kaiser. ${ }^{45}$ Diejenigen, die in den Steinbrüchen oder den Töpferwerkstätten arbeiteten, mussten sowohl an den Fiskus den zehnten Teil ihres Ertrags entrichten als auch an den Eigentümer der Töpfereien bzw. den Eigentümer des Landes, auf dem sich der Steinbruch befand; die verbleibende Summe stand dann dem Arbeiter zu: „Cuncti, qui per privatorum loca saxorum venam laboriosis effossionibus persequuntur, decimas fisco, decimas etiam domino repraesentent, cetero modo suis desideriis vindicando. "46 Außerdem durften sie die von ihnen produzierten Waren nicht selbst verkaufen, denn durch die Pachtverträge wurde der Verkauf allein den conductores zugebilligt, die die Gewinne einstrichen und Abgaben an den procurator des Distrikts abführten. Dieses System

derts n. Chr.“ nicht haltbar, da Inschriften aus der 2. Hälfte des 2. Jahrhunderts n. Chr. existieren. Doch ist der Zeitraum der Datierung dieser Inschriften mit einem großen Intervall angegeben, was die zeitliche Einordnung nicht präzisiert. Nun griffen auch hier - wie schon beim Abbau von Basaltlava - die Bedingungen der Pachtwirtschaft.

${ }^{39}$ GLAUBEN/GRÜNEWALD/GRUNWALD 2009, 150, 155.

${ }^{40}$ P. Oxy. 3595-3597. P. Oxy 3595 stammt aus dem Jahr 243 n. Chr., P. Oxy. 3596 datiert in den Zeitraum zwischen 219 und 255 n. Chr. und P. Oxy. 3597 stammt aus dem Jahr $260 \mathrm{n}$. Chr.

${ }^{41}$ P. Oxy. 3595-3597; vgl. REDKNAP 1999, 53. Nach REDKNAP 1999, 29 gehörten zu einer Töpferei „Tonentnahmegruben, Arbeitsflächen für das Mischen des Tons, eine Werkstatt oder ein Herstellungsbereich, ein überdachtes Lager für gebrannte und ungebrannte Gefäße, ein Brennstofflager [...] sowie ein Wohnhaus für den Töpfer.“ Den ägyptischen coloni wurden keine Wohnungen gestellt (vgl. P. Oxy. 3595-3597).

${ }^{42}$ Vgl. CIL II 5181 II. 27-29; CIL II 5181 II. 32-35; CIL II 5181 II. 40f.; CIL II 1892 II. 1-9; CIL II 1892 II. 37f.; CIL II 1892 II.47-57; CIL II 1892 II. 59-61.

${ }^{43}$ Die so genannte lex Manciana wird in der Inschrift aus Henchir Mettich (CIL XIII 25902; $117 \mathrm{n}$. Chr.) zitiert, die lex Hadriana wird in den Inschriften aus Ain el-Djemala (CIL XIII 25943; hadrianische Zeit) und Ain Ouassel (CIL VIII 26416; Zeit des Septimius Severus) genannt. Die Tablettes Albertini beweisen, dass die beiden leges auch noch in der Spätantike Gültigkeit besaßen, vgl. SCHOLL/SCHUBERT 2004, 81.

${ }^{44}$ Seit der römischen Kaiserzeit wurde mit dem Begriff saltus ,zumeist das große Gut [bezeichnet], das mehrere fundi umfassen konnte“, so KRAUSE 2001, 1266f.; vgl. Dig. 19,1,52pr. Derartige Domänen wurden meist extensiv landwirtschaftlich genutzt. Das geschah vor allem durch die Verpachtung von saltus an coloni.

${ }^{45}$ CIL XIII 25902, col. I 6-20; CIL XIII 26416, col. I; CIL XIII 26416, col. II 13-III 17; CIL XIII 25943, col. III.

${ }^{46}$ Cod. Theod. 10,19,10. 
stellte zwar sicher, dass der römische fiscus die ihm zustehenden Zahlungen erhielt, verhinderte aber die wirtschaftliche Selbstbestimmung der coloni. ${ }^{47}$

Die detaillierte Analyse des territorium metallum von Mayen erlaubt es, auch Rückschlüsse hinsichtlich der administrativen Strukturierung des ager publicus zwischen Vinxtbach und Nahe anzustellen. ${ }^{48}$ Das Pachtwesen - kontrolliert durch procuratores und ihr officium - bildet hier ebenso wie im übrigen imperium Romanum das Fundament der rechtlichen Organisation für die Ausbeutung der Ressourcen und die Eintreibung der Abgaben und Gebühren. ${ }^{49}$ „Item procurator caesaris, non solum cui rerum provinciae cuiusque procuratio mandata erit, sed et is, cui rerum quamvis non omnium. Itaque plures sibi procuratores diversarum rerum rei publicae causa abesse intelleguntur. " ${ }^{50}$ In diesen wirtschaftlichen Strukturen war auch die übrige Verwaltung - u.a. Steuererhebung und Rekrutierung von Soldaten - in einem ager publicus organisiert.

\section{Verwaltungsstrukturen im ager publicus am Mittelrhein}

Das territorium metallum von Mayen liefert erste Anhaltspunkte, wie die Verwaltung im ager publicus zwischen Vinxtbach und Nahe ausgesehen haben könnte. Neben diesem territorium metallum existierten im Raum zwischen Vinxtbach und Nahe weitere derartige Distrikte, die sich auf andere wirtschaftliche Branchen gründeten und deren Hauptorte die Administration übernommen hatten. Somit konnte die Verwaltung auf die Funktionen eines Zentralortes zurückgreifen und auf diese Weise die Rekrutierung von Soldaten sowie die Erhebung der munera organisieren. ${ }^{51}$ Eine

\footnotetext{
${ }^{47}$ GARNSEY 1980, 35. Seit dem Principat war der fiscus die Kasse des Kaisers, „da dieser über den fiscus allein verfügen konnte, besaß er die Möglichkeit, auch mit diesen finanziellen Mitteln einen erheblichen Einfluss auf die Politik zu nehmen“ (SCHNEIDER 1998, 531f.; vgl. R. Gest. div. Aug. 16f.). Zunächst bestand eine strikte Trennung zwischen der Verwaltung des Privatvermögens und den öffentlichen Aufgaben (vgl. Tac. ann. 4,15,2). „Obwohl fiscus und aerarium in der Literatur wiederholt einander gegenübergestellt werden (Tac. ann. 2,47,2; 6,17,1; Plin. paneg. 36; 42,1), bleibt unklar, wie die Funktionen von fiscus und aerarium genau zu unterscheiden sind.“ (SCHNEIDER 1998, 531f.). Denn auch in den fiscus flossen öffentliche Einnahmen, die beispielsweise aus Konfiskationen oder aus Abgaben der Provinzen resultierten (vgl. Suet. Aug. 40,3). „Zwar hält Seneca noch an der Fiktion fest, bei dem fiscus handele es sich um eine private Kasse des princeps (Sen. benef. 7,6,3), aber nach Auffassung des Tacitus war es unwesentlich, ob Geld dem aerarium oder dem fiscus zugewiesen wurde (Tac. ann. 6,2,1). Bei Seneca und Plinius erscheint das Privatvermögen des princeps als patrimonium, bei Plinius steht es neben dem öffentlichen Vermögen, dem imperium (Sen. benef. 7,6,3; Plin. paneg. 50,2; vgl. schon R. Gest. div. Aug. 17,2).“ (SCHNEIDER 1998, 531f.) Laut SCHNEIDER 1998, 531f. wuchs der Einfluss des Kaisers auf die finanziellen Mittel des aerarium stetig an, so dass „eine Trennung beider Kassen schließlich politisch bedeutungslos“ wurde. Letztlich umfasste fiscus „alle Einnahmen und Besitzungen des imperium Romanum, wobei die Entwicklung, die dazu geführt hatte, nicht klar nachgezeichnet werden kann“" (SCHNEIDER 1998, 531f.).

${ }^{48}$ KÖSTNER 2012, 73-85; vgl. HIRT 2010, 49f.

${ }^{49}$ Vgl. CIL XIII 25902, col. I 6-10; CIL XIII 26416, col. I; CIL II 5181, col. I 1-6; CIL II 5181, col. II 14-16. Procuratores der Gallia Belgica und der beiden Germanien: PfLAUM 1961, 1056-1058.

${ }^{50}$ Dig. 4,6,35,2.

${ }^{51}$ Munera beinhalteten zum einen Tätigkeiten in der cura und zum anderen auch Steuern. Dabei wurde „Zwischen den munera patrimonalia, den finanziellen Abgaben, und den munera personalia, die keine finanziellen Leistungen einschlossen, unterschieden; die munera mixta waren dann den curiales, die für die Steuereinziehung verantwortlich waren, auferlegt.“ (CORBIER 2000, 485; vgl. Dig. 50,4,18; 50,4,26). Munera personalia umfassten sowohl Tätigkeiten der cura als auch körperliche Leistungen (vgl. CORBIER 2000, 485).
} 
administrative Strukturierung, die lediglich über die Domänen der conductores funktionierte, war für diese Zwecke nicht ausreichend. ${ }^{52}$

Während demnach die territoria metalla im ager publicus zwischen Vinxtbach und Nahe als untere Ebene der Verwaltung verstanden werden können, können pagi als deren übergeordnete Ebene aufgefasst werden. Dabei wird pagus als Unterteilung des ager publicus aufgefasst und nicht als ,relativ selbständige Unterstämme der civitas“ im gallischen Raum. ${ }^{53}$ Hinweise auf die Dimensionen der kaiserzeitlichen pagi im Raum zwischen Vinxtbach und Nahe können über die frühmittelalterlichen Gaustrukturen, die jedoch erst im 8. und 9. Jahrhundert n. Chr. deutlicher zu Tage treten, gewonnen werden. ${ }^{54}$ Zwischen Vinxtbach und Mosel kann der frühmittelalterliche Mayengau verortet werden, der vermutlich identisch ist mit dem kaiserzeitlichen pagus. Namengebend für dieses Gebiet ist Mayen mit der spätrömischen Befestigungsanlage auf dem Katzenberg. ${ }^{55}$ Womöglich war gerade die herausragende wirtschaftliche Stellung Mayens der Grund für die Benennung des gesamten Gaus. Außerdem sind diese Siedlung und ihr Umland bereits als territorium metallum identifiziert. ${ }^{56}$ Für die vici Cardena/Karden und von Kobern-Gondorf beispielsweise können derartige Distrikte ebenso vermutet werden, wobei ihre wirtschaftliche Grundlage erst noch eruiert werden muss. Doch ist zu vermuten, dass hier vornehmlich Land- und Forstwirtschaft ausgeübt wurden.

Zwischen Mosel und Nahe kann der Trechirgau lokalisiert werden, dessen Hauptort in Trigorium/Treis vermutet wird. ${ }^{57}$ Womöglich spielte aber auch die spätantike befestigte Höhensiedlung von Hochstetten-Dhaun eine zentrale Rolle innerhalb dieses pagus, da hier Mitte des 3. Jahrhunderts $\mathrm{n}$. Chr. ein praefectus arcendis latrocinis (CIL XIII 3689) und seine Truppe stationiert wurden, um gegen eine endemisch aufgetretene Gefährdung durch latrones an der Schnittstelle zwischen Ausoniushöhenstraße, Simmerbach und Nahetal vorzugehen. ${ }^{58}$ Sowohl der Mayengau als auch der Trechirgau gehörten zur Trierer Diözese. ${ }^{59}$ Aus den Gau- bzw. pagus-Strukturen sind jedoch die Kastellvici entlang des Rheins - Antunnacum/Andernach, Confluentes/Koblenz, Boudobriga bzw. Bodobrica/Boppard, Salisio/Bad Salzig und Vosolvia/Oberwesel - herauszunehmen. Im Zuge der Errichtung der Kastelle hatte das Militär die Organisation sowie auch Verwaltungs- und Hoheitsfunktionen übernommen, die sonst von einer civitas oder einem municipium ausgeführt wurden ${ }^{60}$ Ihr Einzugsgebiet aus römischer Zeit stimmt mit dem der frühmittelalterlichen fisci überein. ${ }^{61}$

Dass die frühmittelalterlichen Gaustrukturen tatsächlich als Nachfolger der kaiserzeitlichen pagi angenommen werden können, wird durch die Kontinuität zwischen Spätantike und Frühmittelalter bedingt. ${ }^{62}$ Dabei spielt die so genannte ,frontier society“ bzw. „Grenzkultur“, also die Angleichung zwischen den mehrheitlich germanischen Migranten und den im imperium Romanum lebenden Gallorömern bzw. Rö-

\footnotetext{
52 CÜPPERS 1990, 110; im Gegensatz dazu: vgl. KÖSTNER 2012, 73-85; HIRT 2010, 49f.; RAEPSAETCHARLIER 1999, 312.

${ }^{53}$ GALSTERER 2000, 146f.

${ }^{54}$ HEINEN 1985, 426.

${ }^{55}$ Vgl. EWIG 1976, 438; HunOLD 2011; KÖSTNER 2013A.

${ }^{56}$ KÖSTNER 2012, 73-85.

${ }^{57}$ Vgl. HALFER 1995, 133-157; HeINZELMANN 1995, 9-132.

${ }^{58}$ GILLES 1985, 84; vgl. KÖSTNER 2013B.

${ }^{59}$ Vgl. Vita Lupi ep. Trecensis 11, MGH SRM VII 302, 289.

${ }^{60}$ SOMMER 1988, 457-707; VITTINGHOFF 1994C, 124.

${ }^{61}$ FLACH 1988, 43-52; vgl. u.a. HEYDEN 1956, PAULY 1960/61, 5-27.

${ }^{62}$ EWIG 1976, 412, 438.
} 
mern, eine zentrale Rolle. ${ }^{63}$ Whittakers Konzept der frontier society geht davon aus, dass sich dies- und jenseits des Limes - d.h. sowohl auf dem Gebiet der Germania magna als auch im imperium Romanum - eine Kultur entwickelte, die sich nicht sonderlich voneinander unterschied. ${ }^{64}$ „It was a process of the gradual assimilation of border folk into a culture that was itself changing by adapting to the pressures. So in the end it was unclear who were the barbarians and who were the Romans. "65 Der römische Limes war keine hermetisch abgeriegelte Demarkationslinie, sondern aufgrund der wirtschaftlichen Erfordernisse der am Rhein stationierten Soldaten permeabel, um ökonomische und damit einhergehend auch kulturelle Interaktionen zu ermöglichen. In der Spätantike war diese Interaktion nicht beendet. Mit dieser allmählichen Angleichung zu beiden Seiten des Limes wurde der Grundstein gelegt für ein Fortbestehen römischer Verwaltungsstrukturen am Mittelrhein. ${ }^{66}$ Auch wird davon ausgegangen, dass die kaiserlichen Domänen im frühen Mittelalter in den Besitz der merowingischen Könige übergingen. ${ }^{67}$

Die mittelalterlichen Gaue und fisci am Mittelrhein eröffnen aufgrund der Kontinuität zwischen Spätantike und Frühmittelalter, die auch die Institutionen und Verwaltungsstrukturen umfasste, die Möglichkeit, die administrative Organisation der römischen Kaiserzeit zu erforschen. Die mittelalterlichen Urkunden der Region bieten die Möglichkeit, territoriale Zusammenhänge und Besitzverhältnisse zu erkennen. Womöglich können auf diese Weise Rückschlüsse auf die Verhältnisse der römischen Kaiserzeit gezogen werden. In die beiden territorialen Einheiten Mayengau und Trechirgau, deren Dimensionen wahrscheinlich identisch sind mit denen der kaiserzeitlichen pagi, können kleinere Verwaltungsdistrikte wie z.B. das territorium metallum von Mayen verortet werden.

\footnotetext{
${ }^{63}$ WhitTAKer 1994, 270; FEHR 2010, 710-712. Ein differenzierteres Bild für den Mittelrhein zwischen Spätantike und Frühmittelalter zeichnet SCHMITZ 1997, 195: Er konstatiert in seinen Studien zu frühchristlichen Grabinschriften einen „Unterschied von langer christlicher Tradition in den romanisch geprägten Orten im Rhein- und Moseltal, einer Mischung von Christentum und Diesseitsbezogenheit bei romanisierten Franken und einem schroffen Aufeinanderprallen von christlichem und heidnischem Gedankengut in den Kontaktzonen im ländlichen Raum.“

${ }^{64}$ WHITTAKER 1994, 223. Auch SCHMITZ 1997, 185 bestätigt einerseits das Fortleben römischer bzw. gallorömischer Siedlungen in nachrömischer Zeit (v.a. in den Kastellorten am Rhein, aber z.T. auch in Hunsrück und Eifel); andererseits konnte er eine germanische Besiedlung feststellen.

${ }^{65}$ WHITTAKER 1994, $132 \mathrm{f}$.

${ }^{66}$ FEHR 2010, 42.

${ }^{67}$ Durliat 1997, 517; EwIG 1976, 412; EwIG 1980, 46f.; Cod. Iust. 11,66-68. „Zwischen diesem [= Domäne] und anderem staatlichen Vermögen in kaiserlicher Verfügung gibt es trotz Ansätzen zu einer Scheidung in Staatsgut (fiscus, rationes, largitiones), Krongut (patrimonium, res privata) und persönlichem Gut des Kaisers und seiner Familie (domus augusta, domus divina: Dig. 30,39,8-10) und daraus folgender steuer- und pachtrechtlicher Differenzierung (Cod. Iust. 11,65ff.) keine rechtsprinzipielle und institutionell dauerhafte Grenze, weil der Kaiser nach souveränem Ermessen über alles für alles entscheiden kann (Dig. 43,8,2,4: res fiscales ... quasi propriae et privatae principis sunt).“ (GIZEWSKI 1997, 737f.). Seit den merowingischen Königen umfasste das Reichsgut die Bereiche Krongut, Reichskirchengut und Reichslehengut, wobei nur das Krongut (bzw. Königsgut) als Fiskalbesitz in grundherrlicher Verwaltung oder als wirtschaftliche Ausstattung fungierte (FLACH 1988, 43-52).
} 


\section{Literatur}

\section{Quellenedition}

Cassius Dio

Cassius Dio, Römische Geschichte Bd. IV, übersetzt v. O. Veh, Zürich/München, 1985.

\section{Sekundärliteratur}

AusBÜTtel 2011

F. M. Ausbüttel, Die Gründung und Teilung der Provinz Germania, in: Klio 93, 2011, 392-410.

\section{CÜPPERS 1990}

H. Cüppers, Die Römer in Rheinland-Pfalz, Stuttgart, 1990.

CORBIER 2000

M. Corbier, DNP 3, 2000, 483-486, s. v. munus, munera.

CRAWFORD 1996

M. Crawford, DNP 1, 1996, 251f., s. v. ager publicus.

DURLIAT 1997

J. Durliat, Das Finanzsystem der merowingischen Könige, in: A. Wieczorek /

P. Périn / K. von Welch / W. Menghin (Hrsg.), Die Franken - Wegbereiter Europas Bd. 1, Mainz, 1997, 514-525.

ECK 2004

W. Eck, Köln in römischer Zeit, Geschichte einer Stadt im Rahmen des Imperium Romanum Bd. 1, Köln, 2004.

ECK 2009

W. Eck, Eine römische Provinz, Das augusteische Germanien links und rechts des Rheins, in: S. Burmeister (Hrsg.), 2000 Jahre Varusschlacht, Imperium, Stuttgart, 2009, 188-195.

\section{EWIG 1976}

E. Ewig, Spätantikes und fränkisches Gallien, Gesammelte Schriften (19521973), Bd. 1, München, 1976.

EWIG 1980

E. Ewig, Frühes Mittelalter, Rheinische Geschichte, Bd. 1,2, Düsseldorf, 1980.

FEHR 2010

H. Fehr, Germanen und Romanen im Merowingerreich, Frühgeschichtliche Archäologie zwischen Wissenschaft und Zeitgeschehen, RGA-Ergänzungsbd. 68, Berlin, 2010.

FLACH 1988

D. Flach, Königshof und Fiskus in Andernach, in: F.-J. Heyden (Hrsg.), Andernach, Geschichte einer Stadt, Andernach, 1988, 43-52.

\section{GALSTERER}

H. Galsterer, DNP 9, 2000, 146f., s. v. pagus. 


\section{GARNSEY 1980}

P. Garnsey, Non-Slave Labour in the Roman World, in: P. Garnsey (Hrsg.), Non-Slave Labour in the Greco-Roman World, Cambridge, 1980, 34-47.

\section{GILLES 1985}

K.-J. Gilles, Spätrömische Höhensiedlungen in Eifel und Hunsrück, Trier Zeitschrift-Beihefte Bd. 7, Trier, 1985.

GIZEWSKI 1997

C. Gizewski, DNP 3, 1997, 737f., s. v. Domäne.

GLAUBEN/GRÜNEWALD/GRUNWALD 2009

A. M. Glauben / M. B. Grünewald / L. Grunwald, Mayen am Übergang von Spätantike zu Frühmittelalter, in: O. Wagener (Hrsg.), Der umkämpfte Ort Von der Antike zum Mittelalter, Beihefte zu Mediaevistik Bd. 10, Frankfurt a. M., 2009, 135-156.

\section{HALFER 1995}

M. Halfer, Trigorium, Namenkontinuität im Rhein-Mosel-Dreieck, in: Jahrbuch für Westdeutsche Landesgeschichte 21, 1995, 133-157.

HEINEN 1985

H. Heinen, Trier und das Trevererland in römischer Zeit, Trier, 1985.

\section{HEINZELMANN 1995}

J. Heinzelmann, Der Weg nach Trigorium, Grenzen, Straßen und Herrschaft zwischen Untermosel und Mittelrhein im Frühmittelalter, in: Jahrbuch für Westdeutsche Landesgeschichte 21, 1995, 9-132.

\section{HEYDEN 1956}

F.-J. Heyden, Reichsgut im Rheinland, Die Geschichte des königlichen Fiskus Boppard, Bonn, 1956.

HIRT 2010

A. Hirt, Imperial Mines and Quarries in the Roman World, Organizational Aspects $27 \mathrm{BC}-\mathrm{AD} 235$, Oxford, 2010.

\section{KÖSTNER 2012}

E. Köstner, Stadt, Land, Fluss: Rechtliche Aspekte der Landnutzung in der Eifel nach dem gallischen Krieg, in: M. Grünewald / S. Wenzel (Hrsg.), Römische Landnutzung in der Eifel - Neue Ausgrabungen und Forschungen, RGZM-Tagungen Bd. 16, Mainz, 2012, 73-85.

KÖSTNER 2013A

E. Köstner, Fortifikationen als konservierendes Instrument administrativer Strukturen - am Beispiel Mayens und der Befestigung auf dem Katzenberg, in: O. Wagener (Hrsg.), Burgen und Befestigungen in der Eifel, Von der Antike bis zum Zweiten Weltkrieg, Beih. Mediaevistik (erscheint 2013).

\section{KÖSTNER 2013B}

E. Köstner, Von Räubern und Gendarmen: Der praefectus arcendis latrocinis aus Hochstetten-Dhaun (Kr. Bad Kreuznach), in: B. Edelmann-Singer / H. C. Konen (Hrsg.), Salutationes, Kleine Gaben zur Alten Geschichte und ihrer Rezeption, Festschr. P. Herz (erscheint 2013). 
KRAUSE 2001

J. Krause, DNP 10, 2001, 1266f., s. v. saltus.

\section{LEHNER 1921}

H. Lehner, Ein Tuffsteinbruch des ober- und niedergermanischen Heeres in

Kruft, in: Germania 5, 1921, 130-133.

MANGARTZ 2008

F. Mangartz, Römischer Basaltlava-Abbau zwischen Eifel und Rhein, Monogr. RGZM Bd. 75, Mainz, 2008.

MATIJEVIĆ 2010

K. Matijević, Römische und frühchristliche Zeugnisse im Norden Obergermaniens, Epigraphische Studien zu unterer Mosel und östlicher Eifel, Pharos Bd. 27, Rahden, 2010.

\section{PAULY 1960/1961}

F. Pauly, Der königliche Fiskus von Koblenz, in: Jahrbuch für Geschichte und Kunst des Mittelrheins und seiner Nachbargebiete 12/13, 1960/1961, 5-27.

PFLAUM 1961

H.-G. Pflaum, Les carrières procuratoriennes équestres sous le haut-empire romain, Paris, 1961.

\section{RAEPSAET-CHARLIER 1999}

M.-T. Raepsaet-Charlier, Les instituions municipales dans les Germanies sous le Haut Empire: bilan et questions, in: M. Dondin-Payre / M.-T. RaepsaetCharlier (Hrsg.), Cités, municipes, colonies, Les processus de municipalisation en Gaule et en Germanie sous le Haut Empire romain, Paris, 1999, 271-354.

RATHMANN 2003

M. Rathmann, Untersuchungen zu den Reichsstraßen in den westlichen Provinzen des Imperium Romanum, Bonner Jahrbücher-Beiheft Bd. 55, Mainz, 2003.

\section{REDKNAP 1999}

M. Redknap, Die römischen und mittelalterlichen Töpfereien in Mayen, in: H.H. Wegner (Hrsg.), Berichte zur Archäologie an Mittelrhein und Mosel Bd. 6., Trierer Zeitschrift-Beiheft Bd. 24, Trier, 1999, 11-402.

\section{RÖDER 1957}

J. Röder, Die antiken Tuffsteinbrüche der Pellenz, in: Bonner Jahrbücher 157, 1957, 213-271.

SCHAAFF 2010

H. Schaaff, Steine für das römische Reich, $\mathrm{Zu}$ den Anfängen des antiken Steinbruch- und Bergbaureviers zwischen Eifel und Rhein, in: Archäologisches Korrespondenzblatt 40, 2010, 265-272.

\section{SCHAAFF 2012}

H. Schaaff, Steine für den römischen Städtebau - Die Grube Idylle bei Kruft und Kretz, in: Der Anschnitt 64, 2012, 2-17. 


\section{SCHARF 2005}

R. Scharf, Der Dux Mogontiacensis und die Notitia Dignitatum, Eine Studie zur spätantiken Grenzverteidigung, RGA-Ergänzungsbd. 80, Berlin/New York, 2005.

\section{SCHMITZ 1997}

W. Schmitz, Zur Akkulturation von Romanen und Germanen im Rheinland, in: Das Altertum 43, 1997, 177-202.

\section{SCHNEIDER 1998}

H. Schneider, DNP 4, 1998, 531f., s. v. fiscus.

\section{SCHOLL/SCHUBERT 2004}

R. Scholl / C. Schubert, Lex Hadriana de agris rudibus und Lex Manciana, in: Archiv für Papyrusforschung 50/1, 2004, 79-84.

\section{SOMMER 1988}

C. S. Sommer, Kastellvicus und Kastell, in: Fundberichte Baden-Württemberg 13, 1988, 457-707.

\section{VITTINGHOFF 1994A}

F. Vittinghoff, Die politische Organisation der römischen Rheingebiete, in: W. Eck (Hrsg.), Civitas Romana, Stadt und politisch-soziale Integration im Imperium Romanum der Kaiserzeit, Stuttgart 1994, 66-88 (erschienen in: Convegno internazionale Renania Romana, Rom, 1976, 73-94).

\section{VITTINGHOFF 1994B}

F. Vittinghoff, Zur Entwicklung der städtischen Selbstverwaltung: einige kritische Anmerkungen, in: W. Eck (Hrsg.), Civitas Romana, Stadt und politischsoziale Integration im Imperium Romanum der Kaiserzeit, Stuttgart, 1994, 218-249 (erschienen in: F. Vittinghoff (Hrsg.), Stadt und Herrschaft, Römische Kaiserzeit und hohes Mittelalter, München, 1982, 107-146.).

\section{VITTINGHOFF 1994C}

F. Vittinghoff, Das Problem des ,Militärterritoriums' in der vorseverischen Kaiserzeit, in: W. Eck (Hrsg.), Civitas Romana, Stadt und politisch-soziale Integration im Imperium Romanum der Kaiserzeit, Stuttgart, 1994, 124-139 (erschienen in: Atti del convegno internazionale sul tema: I diritti locali nelle province Romane con particulare riguardo alle condizione giuridiche del suolo, Academia Nazionale die Lincei, Problemi attuali di scienza e di cultura, Quaderno 194, Rom 1974, 109-124.).

\section{WHITTAKER 1994}

C. R. Whittaker, Frontiers of the Roman Empire, A Social and Economic Study, Baltimore, London, 1994.

\section{WITTEYER 1999}

M. Witteyer, Mogontiacum - Militärbasis und Verwaltungszentrum, Der archäologische Befund, in: F. Dumont / F. Scherf / F. Schütz (Hrsg.), Mainz, Die Geschichte der Stadt, Mainz 1999, 1021-1058. 
Frankfurter elektronische Rundschau zur Altertumskunde 19 (2012)

\section{Kontakt zur Autorin:}

Dr. Elena Köstner

Lehrstuhl für Alte Geschichte

Fakultät für Philosophie, Kunst-,

Geschichts- und Gesellschaftswissenschaften

Universität Regensburg

E-Mail: Elena.Koestner@geschichte.uni-regensburg.de 